DOI: 10.17234/SRAZ.65.24

UDK: 821.133.1.09 Sardou, V.

UDK: 821.133.1.091:821.163.42

Original scientific paper

Reçu le 7 septembre 2020

Accepté pour la publication le 25 novembre 2020

\title{
La réception de Victorien Sardou (1831-1908) en Croatie ${ }^{1}$
}

\section{Crijeta Pavlović}

Faculté de Philosophie et Lettres de l'Université de Zagreb

cvijeta.pavlovic@ffzg.hr

Victorien Sardou (1831-1908), auteur dramatique français, a joui d'un succès remarquable et d'une prompte réception dans la littérature et le théâtre croates du XIX ${ }^{\mathrm{e}}$ siècle, en concoctant des pièces bien faites versant dans la satire sociale. La présente analyse révèle les particularités qui ont rendu possible une convergence du style de Victorien Sardou, des opinions et des concepts exprimés par cet auteur dans son œuvre, avec le contexte culturel et poétique croate. Le machinisme dramatique, une intrigue bien ficelée, les «joujoux » dramatiques de Sardou et de son « maître » Eugène Scribe sur la scène croate contribuent à l'identification et la compréhension des tendances réalistes au sein de la littérature croate de la deuxième moitié du XIXe siècle, laquelle est appelée « époque de Šenoa ».

Mots-clés : Victorien Sardou, réception, époque de Šenoa, machinisme dramatique, réalisme

Une approche comparatiste suppose une étude de la réception de la littérature et de la culture françaises en Croatie, englobant des questions relevant de la périodisation, de l'esthétique, de la poétique, voire du canon de la littérature de la sphère occidentale ou, plus largement, mondiale. L'objectif de la présente étude consiste à mettre en lumière le rôle qu'a joué dans l'histoire de la littérature croate l'œuvre de Victorien Sardou (1831-1908) à des époques littéraires particulièrement turbulentes, qui ont vu se multiplier les styles artistiques : romantisme à son déclin, réalisme, art pour l'artisme et mouvement parnassien, naturalisme, jusqu'au modernisme (avec la décadence, le symbolisme, l'impressionisme, l'art nouveau ; autant de tendances qui - quant à la nomenclature de la littérature

Cet article est financé par la Fondation croate pour la science (Hrvatska zaklada za znanost) pour le projet Les Fondements économiques de la littérature croate - Ekonomski temelji hrvatske književnosti (HRZZ IP-2016-06-2613). La recherche qui a précédé sa rédaction a été conduite aux archives du Département d'histoire du théâtre croate de l'Institut d'histoire de la littérature, du théâtre et de la musique de l'Académie croate des sciences et des arts. 
mondiale ou générale - peuvent être conçues comme les différentes manifestations de l'esprit de l'esthétisme de leur temps).

Il y a plusieurs mots qui sautent aux yeux dans les discours sur Victorien Sardou : un de ces mots est le succès, l'autre est le profit, et le troisième mot est : l'oubli. Ce modèle est l'un des plus fréquents dans le cours de l'histoire de la littérature et de l'art. Il convient, pour en scruter les rouages, de vérifier dans quelle mesure l'économie exerce, dans le cadre de la culture et de la littérature mondiale, une influence sur le processus de consécration de l'auteur. A l'aube du XXI ${ }^{\mathrm{e}}$ siècle se sont succédées plusieurs études et monographies consacrées à cet auteur, signées, entre autres, par Isabelle Moindrot (2001), Dieter Schickling (2004), Guy Ducrey et Walter Zidaric (2007), John Henry Stape et Allan Simmons (2007), Marion Pouffary (2009), Blanche Roosevelt et Evan Baker (2009), ou encore Michele Girardi (2010), pour ne citer qu'eux.

Malheureusement, Sardou ne figure pas dans le dictionnaire des écrivains étrangers Leksikon stranih pisaca (2001), bien que s'y trouvent ses contemporains et confrères Émile Augier, Alexandre Dumas fils, ainsi que leur maître Eugène Scribe. La décision d'omettre Sardou de cette publication croate de référence trouve son fondement et sa justification dans le troisième tome de l'histoire de la littérature mondiale consacré à la littérature française (Povijest svjetske književnosti, knj. 3, Francuska knjižeonost), dans le chapitre abordant le théâtre post-romantique sous le titre «U znaku građanskog kazališta » (« sous le signe du théâtre bourgeois »), où Helena Mandić-Pachl reproduit des propositions et thèses répandues : le théâtre de Sardou ne peut pas survivre à son public, à savoir à la foule pour laquelle il était écrit ; partageant ce destin avec Augier et Dumas fils, il n’a pas dépassé Scribe, préoccupé qu'il était avant tout par les effets, la scène, voire même la salle concrète et réelle (Mandić-Pachl 1982 : 551).

Cependant, Sardou est paradoxalement l'auteur dramatique le plus joué, le plus mis en scène et le plus représenté dans les théâtres croates au XIX ${ }^{\mathrm{e}}$ siècle (Hećimović 1990, Batušić 1978 : 253, 268, 298, 378, 449). Si on y joint les compterendus et les articles qui lui sont consacrés dans les revues et périodiques littéraires et culturels (Lapenda 2008, Šimundža 1993), on obtient une imposante quantité de mentions dans les publications de l'époque, à la mesure du nombre de pièces de Sardou traduites et montées en Croatie : Posljednje ljubavno pismo (Les Pattes de mouche) ; Dobri prijatelji ainsi qu'une autre traduction sous le titre de Prisni prijatelji (Nos intimes) ; Dora; plusieurs traductions, sous plusieurs titres, de Divorçons (Raskrstimo se, Raziđimo se!, Ciprienne, Cyprienna, Cyprienne) ; Domovina (La Patrie!) ; Rabagas ; Nervozni (Les Gens nerveux) ; Bogomoljci (Séraphine) ; Fernanda (Fernande); Dobričine seljaci (Nos bons villageois) ; Moderni malograđani (Les Bourgeois de Pont-Arcy) ; Daniel Rochat ; Odeta (Odette) ; Georgetta (Georgette) ; Prevrtljivost (La Papillonne) ; Fedora (Fédora) ; Stari mladići (Les vieux garçons) ; Madame SansGêne ; Andrea (Andréa); Gismonda (La Duchesse d'Athènes) ; Spiritizam (Spiritisme) ; Tosca (La Tosca) ; Ferreol (Ferréol) ; Margerita (Les Ganaches) ; Thermidor ; Marcella (Marcelle); Theodora (Théodora); Vještica (La Sorcière); Obitelj Benoîton (La Famille Benoîton). La suite de cette liste dépasse les limites du XIX ${ }^{\mathrm{e}}$ siècle et s'étend aux débuts du XXe siècle (Hećimović : 1990). 
A l'instar des Anglais, qui peuvent à propos de Sardou citer des remarques et pensées écrites par les célèbres plumes de George Bernard Shaw ou Oscar Wilde, les Croates disposent au sujet de cet auteur, de textes signés par plusieurs noms importants de la critique, de la littérature et de sa théorie : August Šenoa, Ivan Perkovac, Josip Pasarić, Janko Ibler, Jovan Hranilović, Dinko Politeo etc.

Pour les critiques de théâtre croates, Sardou avait aussi une réputation de symbole et de type dramatique, car ils lui attribuent (aussi bien qu'à Dumas) des modèles d'écriture, en soulignant qu'il expose dans ses textes mille aspects du même sujet (Šimundža 1993 : 49). Il apparaît par ailleurs, au vu du répertoire des troupes en tournée, qu'à la faveur des choix des acteurs et des metteurs en scène, les auteurs les plus représentatifs de la culture, de la littérature et du théâtre français du XIX ${ }^{\mathrm{e}}$ siècle figuraient à l'affiche en compagnie de prestigieux auteurs européens tels que Shakespeare et Wilde, Goldoni, Ibsen, Gogolj, Tchekhov et Gorki (Lapenda 2008 : 34-42, 169-170). Sardou côtoyait donc dans les répertoires aux côtés d'auteurs de qualité, consacrés.

L'analyse chronologique des compte-rendus sur Sardou et son œuvre révèle que le périodique Vienac informait régulièrement sur les événements en France et renseignait également assidûment ses lecteurs sur le répertoire des théâtres et la qualité des productions (Šimundža 1993 : 49-160; Hećimović 1990; Batušić 1978; Batušić 1992).

Les critiques croates du XIX ${ }^{\mathrm{e}}$ siècle se montrèrent d'abord réservés. Cependant, après la «consécration » de Sardou par son entrée à l'Académie française, qui vint couronner ses succès auprès du public croate et dans le monde entier, les critiques se virent contraints d'admettre que, même si demeuraient les remarques à son sujet, force était de les nuancer en prenant en considération l'incontestable goût du public pour Sardou et l'indéniable gloire de ce dernier, témoignages de l'esprit de son temps. Un semblable développement du processus de réception est déjà constaté scientifiquement pour un autre célèbre écrivain français, à savoir Jules Verne (Pavlović 2018 :38-57). Les fondements de toutes ces critiques reposent sur le raisonnement selon lequel les pièces de Sardou dépassaient en qualité celles du répertoire allemand, qui était dominant à Zagreb. Ainsi Sardou figurait-il dans le paysage littéraire comme une des armes dans la lutte culturelle croate contre la prédominance allemande (et autrichienne) : Schikaneder, Raupach, Raimund, von Kotzebue vs. Sardou, Feuillet, Ponsard, Augier (Šenoa 1964 [1866] : 524- 567 [120]; Batušić 1978 : 253; Pavlović 2005, Pavlović 2006 et al.).

Son succès en Croatie fut également conforté par le travail des traducteurs de ses pièces, parmi lequels figuraient des artistes, des écrivains, des rédacteurs, des acteurs, tels Dimitrija Demeter, August Šenoa, Josip Eugen Tomić, Stjepan Miletić, Tito Strozzi, Marija Ružička-Strozzi, Branko Gavella et d'autres.

Pour se bâtir, cette idée s'étaye à des éléments clés, à savoir des hommes mais aussi des femmes, notamment des actrices : Sarah Bernhardt, Fanny Davenport, Gloria Swanson puis, un demi-siècle plus tard, Sophia Loren, etc. En Croatie c'est, entre autres, Marija Ružička-Strozzi. Les pièces Fédora, Théodora, La Tosca, Cléopâtre, Gismonda et Spiritisme ont été écrites pour l'actrice la plus célèbre de son temps, Sarah Bernhardt. Mais la réussite du texte est également soutenue par la 
musique (Fédora, 1882 ; Madame Sans-Gêne, 1893 ; la Tosca, 1900, avec beaucoup d'autres opéras dès 1886, de comédies musicales dès 1907 et d'adaptations cinématographiques dès 1912). Le premier opéra inspiré d'un texte de Sardou donné en Croatie était Fédora, dont la première représentation eut lieu en 1903 (Hećimović 1990 : 1-2).

Quant à l'oubli, processus naturel dans la vie littéraire, théâtrale et culturelle (Bourdieu 1998 ; Šoštarić 2017), il n’a en fait pas totalement balayé Sardou, car cet auteur donc continue de vivre à travers l'opéra, avec notamment la Tosca de Puccini. Il s'agit bien sûr d'une musique merveilleuse, mais l'opéra doit aussi sa longévité à son sujet, particulièrement poignant et indémodable. En témoigne la décision de présenter cet opéra dans le cadre du programme de la Capitale Européenne de la Culture Rijeka 2020. Dans son annonce, Marin Blažević, l'intendant du Théâtre Ivan pl. Zajc, Théâtre national croate de Rijeka, souligne combien le sujet - les passions et le conflit éternel qui déchirent les personnages - de cette pièce politique très aisément transposable, est efficace pour dénoncer tous les totalitarismes (au $\mathrm{XIX}^{\mathrm{e}}$ siècle comme au $\mathrm{XX} \mathrm{e}^{\mathrm{e}}$ siècle, jusqu'au fascisme et au communisme, etc.) et, au-delà, tous les visages du pouvoir.

Ainsi, sur la page actuelle de l'Encyclopédie Cosmovision - Imago Mundi dédiée à Victorien Sardou, l'œuvre de cet auteur est illustrée par le dialogue " les Parisiens aux champs », tiré de la comédie Nos bons villageois ${ }^{2}$. Ce n'est pas un hasard si August Šenoa choisit de traduire cette pièce en croate : Šenoa avait le goût du réalisme, ainsi que de tout ce qui est « classique ». Cette citation illustre fort bien les paramètres actuels et contemporains sur les questions de la propriété et du propriétarisme : il y évoque la tension entre la province et la capitale, le village et la métropole, les villageois et les bourgeois, mais aussi des problèmes qui opposent les autochtones et les nouveaux venus sur fond de privilèges. A l'époque de Šenoa, à savoir dans la deuxième moitié du XIX ${ }^{\mathrm{e}}$ siècle, les idées de Sardou sur la propriété et sur la tension entre le centre et la périphérie, exprimées en dialogues réalistes bien tournés suscitaient aussi de grands éclats de rire dans le public croate. Il est dommage qu'on évite de nommer l'époque qui couvre le milieu et la seconde moitié du XIXe siècle par une nomenclature appropriée et en correspondance avec les autres époques littéraires et théâtrales (Mandić-Pachl 1982 : 548). La solution émerge, soutenue par les conclusions de Bourdieu (1998) et Itamar Even-Zohar (1990) sur les règles de l'art, le champ littéraire, l'économie, les polysystèmes, à travers le centre et la périphérie, jusqu'à la traductologie. Le réalisme enforcit et s'affirme sans se dérober au langage, or le langage est au centre des pièces que signe Victorien Sardou. La pièce de Sardou dans la traduction d'August Šenoa (1870) fut présentée au public durant de longues années, de 1870 à 1897, soit bien après la mort de Šenoa. Les raisons du succès de la réception de Victorien Sardou en Croatie et dans le monde entier renforcent l'idée que cette époque cultivait le goût et l'esprit réaliste.

2 http://www.cosmovisions.com/Sardou.html 
Pour finir, je tiens à dédier cette analyse dans le champ de la littérature comparée aux professeurs de la Chaire de langue et littérature françaises qui ont apporté leur contribution à l'étude de la littérature comparée, en particulier G. Vidan, I. Šafranek, A. Kovačec, J. Tarle et N. Ivić.

\section{Bibliographie:}

Baker, Evan (2009) Sardou and Sardoodledom, Puccini and Tosca. Programme Notes, San Francisco Opera. http://sfopera.com/o/273.asp (11/11/2019).

Batušić, Nikola (1978) Povijest hrvatskoga kazališta, Zagreb: Školska knjiga.

Batušić, Nikola (dir.) (1992) Hrvatsko narodno kazalište u Zagrebu, Zagreb: Hrvatsko narodno kazalište, Tiskara Puljko.

Bourdieu, Pierre (1998). Les Règles de l'art: genèse et structure du champ littéraire, Paris: Édition du Seuil.

Ducrey, Guy (dir.) (2007). Victorien Sardou, un siècle plus tard, Strasbourg: Presses universitaires de Strasboug.

Even-Zohar, Itamar (1990). Polysystem studies, in: Poetics Today, v. 11, n. 1, pp. 1-262.

Girardi, Michele (dir.) (2000) Tosca 18001900 2000. Catalogue of the exhibition held at the Museo Nazionale di Villa Guinigi. Luca, 2-27 February 2000 http:// www.puccini.it/scientifica/MostraTosca.pdf (11/11/2019).

Hećimović, Branko (1990). Repertoar hrvatskih kazališta, knj. 1, knj. 2, Zagreb: Globus. Jugoslavenska akademija znanosti i umjetnosti; knj. 3, Zagreb: Hrvatska akademija znanosti i umjetnosti. AGM.

Lapenda, Stjepan (2008). Francuski duh u hrvatskoj periodici u Dalmaciji 1844. - 1918. = La présence française dans les périodiques croates en Dalmatie 1844-1918, Split: Književni krug.

Mandić-Pachl, Helena (1982) Kazalište nakon romantizma, in: Povijest svjetske književnosti, knj. 3, Francuska književnost, Zagreb: Liber, Mladost, pp. 548-587.

Moindrot, Isabelle (dir.) (2011). Victorien Sardou. Le théâtre et les arts, Rennes: Presses universitaires de Rennes, https://books.openedition.org/pur (11/13/2019).

Pavlović, Cvijeta (2005). Veze i utjecaji. Zaključak, in: Priča u pjesmi, Zagreb: Disput, pp. 181-215.

Pavlović, Cvijeta (2006). Šenoina poetika prevođenja. Zagreb: Matica hrvatska.

Pavlović, Cvijeta (2018). August Šenoa i Jules Verne: Percepcija pučke ili popularne i beletrističke književnosti u 19. stoljeću, in: Dani Hvarskoga kazališta. Pučko i popularno II, t. 44 [éd. Boris Senker], Zagreb - Split: Hrvatska akademija znanosti i umjetnosti, Književni krug, pp. 38-57

Roosevelt, Blanche (2009). Victorien Sardou. Charleston: BiblioLife.

Sardou, Victorien (1878). Discours de réception de Victorien Sardou le 23 mai 1878 http://www.academie-francaise.fr/discours-de-reception-de-victorien-sardou (11/15/2019).

Sardou, in : Imago mundi. Encyclopédie gratuite en ligne, http://www.cosmovisions. com/Sardou.html. (11/13/2019). 
Schickling, Dieter (2004) Fictional Reality: Musical and Literary Imagery in the Toscas of Sardou and Puccini, in : Deborah Burton, Susan Vandiver Nicassio and Agostino Ziino [éd.], Tosca's Prism: Three Moments of Western Cultural History. Northeastern University Press. https://books.google.com/ books?id=cQ_cpUToSKMC (11/11/2019).

Stape, John Henry et Simmons, Allan (2007) Tosca's Kiss: Sardou, Puccini and The Secret Agent in : Stape and Simmons [éd.], Rodopi, pp. 106-116. https://books. google.com/books?id=eOqppWKQuRcC\&pg=PA108\&dq=Tosca+dishes+Ber nhardt\&hl=en\&ei=8RYzTNH2JIbGIQePmJy-Cw\&sa=X\&oi=book_result\&ct= reult\&resnum $=5 \&$ ved=0CEEQ6AEwBA\# $\mathrm{v}=$ onepage\&q\&f=false $(11 / 11 / 2019)$

Šenoa, August (1964) O hrvatskom kazalištu, t. IX, in: Sabrana djela [éd. Slavko Ježić], t. I -XII, Zagreb: Znanje, pp. 524-567.

Šenoa, August, trans., 1870: V. Sardou: Dobričine seljaci. Igrokaz u 5 (4) činah, manuscript 449. Zagreb: le Département de l'histoire du théâtre croate dans l'Institut de l'histoire de littérature, théâtre et musique de l'Académie croate des sciences et des arts (Hrvatska akademija znanosti i umjetnosti).

Šimundža, Drago (1993). Francuska književnost u „Viencu“, Split: Književni krug. Šoštarić, Sanja (2017) Od slave do zaborava. Recepcija književnokritičkoga i romanesknoga opusa Paula Bourgeta, Zagreb: Ex libris.

\section{Recepcija Victoriena Sardoua (1831. - 1908.) u Hrvatskoj}

Victorien Sardou (1831. - 1908.), francuski dramatičar, koji je glasovitu formu dobro skrojenih komada zaokrenuo prema društvenoj satiri, uživao je velik europski i svjetski uspjeh te imao promptnu recepciju u hrvatskoj književnosti i kazalištu XIX. stoljeća. Analiza otkriva osobitosti hrvatske kulture i kompatibilnost hrvatskih poetskih i kulturnih uvjeta sa stilskim smjernicama, mišljenjem, pogledima i konceptima djela Victoriena Sardoua. Dramski mašinizam, dobro skrojeni zapleti, dramske ,igračke“ Sardoua i njegova „učitelja“ Eugènea Scribea tijekom hrvatskih uprizorenja predstava pomažu da se shvate i da se kritički imenuju realističke tendencije u hrvatskoj književnosti druge polovice XIX. stoljeća. To je razdoblje hrvatske književnosti poznato i pod nazivom „Šenoino doba“. Sardouovi omjeri stvarnosti u epohi realizma još uvijek su poticajni za umjetničko i znanstveno promišljanje stvarnosti i njezinih vrjednosnih polja. Ovo istraživanje, usredotočeno na ekonomske temelje književnosti, daje odgovor na moguće imenovanje stila razdoblja u kojemu vladaju dobro skrojeni komadi (pièces bien faites), a s druge strane i imenovanje epohe hrvatske književnosti druge polovice XIX. st u europskom kontekstu.

Ključne riječi: Victorien Sardou, recepcija, Šenoino doba, dramski mašinizam, realizam 